\title{
Characterization of Kidney Disease in Adults' Patients Using Ultrasonography
} Abdulrahman M. B. Arbab ${ }^{1 *}$, Shima I. Ali ${ }^{2}$, M.A.H. Rushdi ${ }^{3}$, M. E. M. Gar-Elnabi ${ }^{1}$

${ }^{1}$ Sudan University of Science and Technology College of Medical Radiologic Science, P.O. Box 1908, Khartoum, Sudan

${ }^{2}$ Faculy of Radiological Sciences and Medical Imaging, Alzaiem Alazhari University, Khartoum, Sudan

${ }^{3}$ Sudan Atomic Energy Commission, Khartoum, Sudan

DOI: $10.36347 /$ sjams.2021.v09i03.015

| Received: 22.02.2021 | Accepted: 03.03.2021 | Published: 08.03.2021

*Corresponding author: Abdulrahman Arbab

Abstract

Original Research Article

The aim of this study to characterization of kidney disease in adults' patients using ultrasonography, the data of this study was collected from 200 adults' patients both gender suffering from renal disorders and referred to ultrasound department in east coast kalba hospital - Sharjah province United Arab Emirates in period from December 2017 up to July 2018. Classification score matrix generated by linear discriminate analysis and the overall classification accuracy of renal disorders $95.4 \%$, were the classification accuracy of normal 98.6\%, HT 94\%, and MHT 93.8\%, while the DM showed a classification accuracy of $92.9 \%$. Figure 1. Error bar plot shows the BMI of normal patients, hypertensive, mild hypertensive and diabetic patient's. The BMI is low in normal patients while it is high in hypertensive and diabetic patients and moderate in mild hypertensive patients. A diagram shows the depth of the left kidney in normal patients, hypertensive, mild hypertensive and diabetic patients. The depth is medium in normal patients, while it is smaller in hypertensive patients; the depth is bigger in mild hypertensive patients and little less in diabetic patients. The end diastolic velocity of the right kidney in normal patients, hypertensive, mild hypertensive and diabetic patients In normal patients the end diastolic is low, but lower in mild hypertensive patients; in diabetic patients it is higher than in .mild hypertensive patients, but less high than in hypertensive patients. It is very low in normal patients, high in hypertensive and mild hypertensive patients and little less in diabetic patients.

Keywords: kidney disease, hypertensive, diabetic, Ultrasonography.

Copyright $\odot$ (C)21 The Author(s): This is an open-access article distributed under the terms of the Creative Commons Attribution 4.0 International License (CC BY-NC 4.0) which permits unrestricted use, distribution, and reproduction in any medium for non-commercial use provided the original author and source are credited.

\section{INTRODUCTION}

Ultrasound is a technique commonly used to evaluate the structure of the kidney and urinary Collecting systems $[1,2]$. Serial sonographic measurements of renal length are often used to evaluate whether the kidneys are growing appropriately; lack of normal renal growth raises the suspicion that the kidney may be undergoing chronic or repeated insults, such as from vesicoureteral reflux or pyelonephritis [3-5]. Although ultrasound has an advantage over other imaging techniques in that it uses no ionizing radiation and does not require sedation, its accuracy in evaluating renal length for the purposes of determining appropriate renal growth is limited. Ultrasound measurement of renal length is frequently used as an indicator of the chronicity of renal disease, with avajue of $9 \mathrm{~cm}$ or less considered to indicate Irreversible disease [6]. It is also an important factor in the decision to undertake renal biopsy, as knowledge of the histology of shrunken kidneys in chronic renal failure is frequently UN helpful in subsequent treatment and the Complication rate following biopsy is increased in shrunken kidneys [7]. It is therefore important that Sonographic renal length measurements are consistent, both for replicate measurements by a single ultra-sonographer and by different ultra-sonographers.

\section{Screening indications}

Multiple guidelines recommend that patients with diabetes or hypertension be screened annually for CKD. Furthermore, patients with other risk factors, including cardiovascular disease, older age, history of low birth weight, obesity, and a family history of CKD, warrant Consideration for screening [8-10]. The U.S. Preventive Services Task Force concluded that the evidence is insufficient to assess the balance of benefits and harms of routine screening for CKD in asymptomatic adults [11]. The American College of Physicians and the American Academy of Family Physicians recommend against screening for CKD in asymptomatic adults without risk factors $[12,13]$. 
Abdulrahman M. B. Arbab et al; Sch J App Med Sci, Mar, 2021; 9(3): 372-377

\section{Screening tests}

Screening for CKD includes measurement of serum creatinine, estimation of GFR using a serum creatinine-based equation, measurement of the urine albumin/creatinine ratio, and urinalysis [8]. Urinalysis has a high sensitivity for heavy proteinuria (greater than $300 \mathrm{mg}$ per 24 hours, as estimated from the spot urine protein/creatinine ratio) but may not detect clinically significant lower levels (30 to $300 \mathrm{mg}$ ) [14]. Because albumin is the predominantly filtered glomerular protein, initial proteinuria evaluation using the spot urine albumin/creatinine ratio obtained from an early morning sample is recommended $[8,15,16]$. Timed 24hoururine collections are no longer recommended as an initial diagnostic tool because of the potential for inadequate collection, inconvenience to patients, and the lack of diagnostic advantage over the urine albumin/creatinine ratio.

\section{Methodology}

The data of this study was collected from 200 adults' patients both gender suffering from renal disorders and referred to ultrasound department in east coast kalba hospital - Sharjah province United Arab Emirates in period from December 2017 up to July 2018.

Tools and equipment's: Ultrasound system general electric GE. Transducer: highest frequency curved linear array probe possible, start with $5 \mathrm{MHZ}$ and work down to 2 or $3 \mathrm{MHZ}$ for larger patients with color and doppler capabilities. A high sweep speed will improve accuracy of the measurements taken to the spectral trace.
The patient's variables were age, gender, kidney volume and resistance index of the right and left kidneys.

\section{Scanning technique}

The patient should be lie supine, for the right kidney have the patient lie supine and place the probe in the right lower intercostal space in the mid axillary line. And the liver as your acoustic window and aim the probe slightly posteriorly toward the kidney. Gently rock the probe up and down or side to side to scan the interior kidney. Obtain longitudinal (long axis) and transverse (short axis) views.

For the left kidney the patient has lie supine or in the right lateral decubiti position, place the prob in the lower intercostal space on the posterior axial line. The placement will be more cephement and posterior than when visualizing right kidney, and again rock the probe to scan the entire kidney to obtain longitudinal and transverse view.

Assessing the arteries within the kidney parenchyma to assess any alteration in the waveforms. The RI should be low resistance. The acceleration time (AT) should be $<70 \mathrm{msec}$. the probe is slowly moved superior and inferior to search for additional renal arteries. Any vessels identified must be traced to the kidney and confirm their identity. The kidneys will be atrophy with chronic renal failure and the length should be $<9 \mathrm{~cm}$, the RI $>0.8 \mathrm{~cm}$ for untreatable medical renal disease.

\section{RESUlts}

Table-1: Showed the classification accuracy of the Predicted Group Membership for the four classes using linear discriminant analysis

\begin{tabular}{|l|l|l|l|l|l|l|}
\hline \multirow{2}{*}{ Classes } & \multicolumn{3}{|l|}{ Predicted Group Membership } & \multirow{2}{*}{ Total } \\
\cline { 3 - 6 } & Normal & HT & MHT & DM & 100.0 \\
\hline \multirow{4}{*}{$\%$} & Normal & 98.6 & .7 & 0.0 & .7 & 100.0 \\
\cline { 2 - 6 } & HT & 4.5 & 94.0 & 0.0 & 1.5 & 100.0 \\
\cline { 2 - 6 } & MHT & 6.2 & 0.0 & 93.8 & 0.0 & 100.0 \\
\cline { 2 - 5 } & DM & 4.1 & 2.0 & 1.0 & 92.9 & \\
\hline $95.4 \%$ of original grouped cases correctly classified.
\end{tabular}




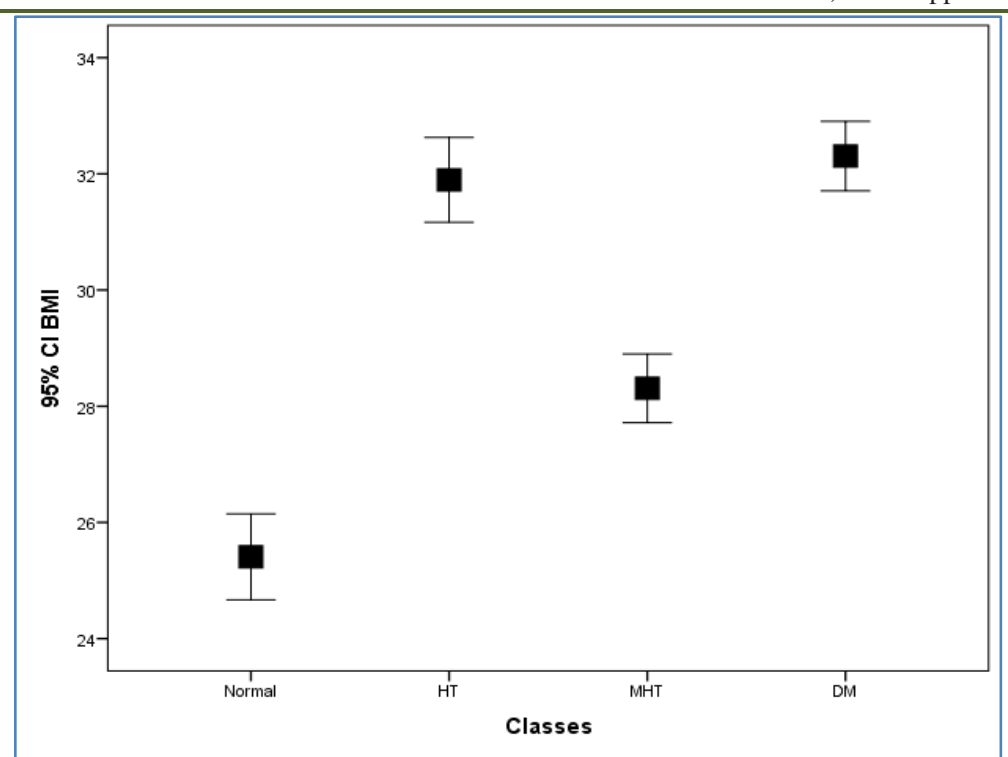

Fig-1: Error bar plot shows the BMI of normal patients, hypertensive, mild hypertensive and diabetic patients. The BMI is low in normal patients while it is high in hypertensive and diabetic patients and moderate in mild hypertensive patients.

TTFF

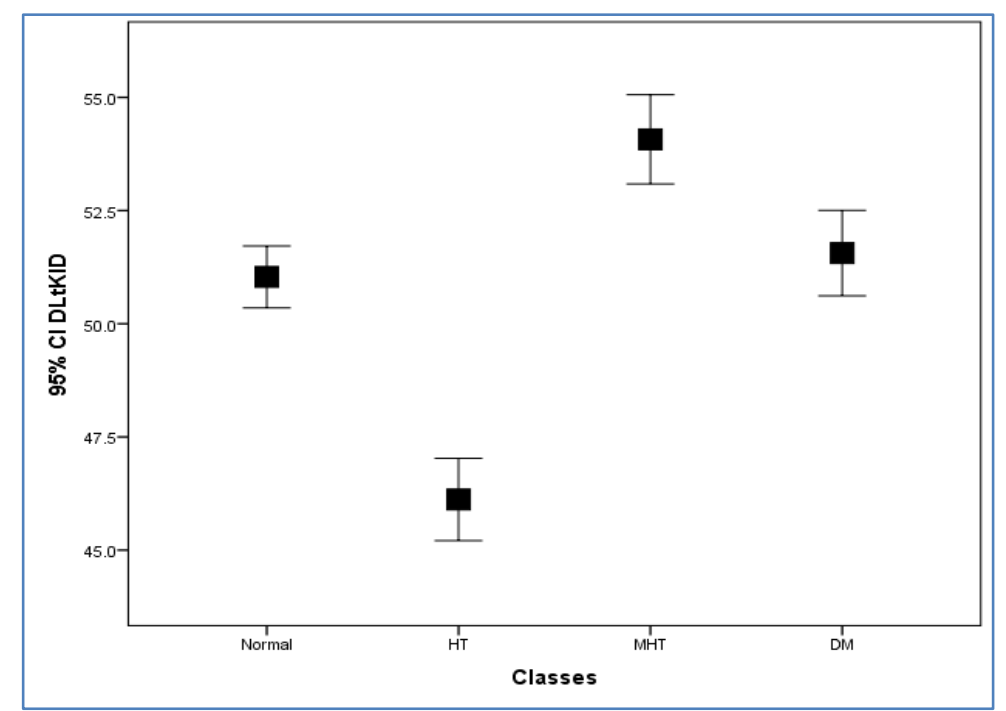

Fig-2: This diagram shows the depth of the left kidney in normal patients, hypertensive, mild hypertensive and diabetic patients. The depth is medium in normal patients, while it is smaller in hypertensive patients; the depth is bigger in mild hypertensive patients and little less in diabetic patients,

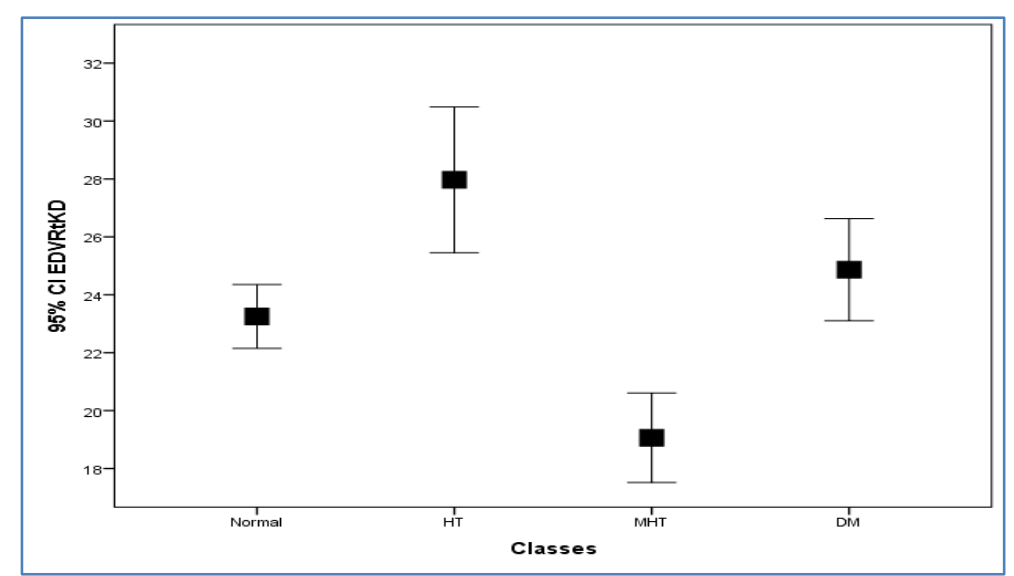

Fig-3: Shows the end diastolic velocity of the right kidney in normal patients, hypertensive, mild hypertensive and diabetic patients. In normal patients the end diastolic is low, but lower in mild hypertensive patients; in diabetic patients it is higher than in .mild hypertensive patients, but less high than in hypertensive patients 


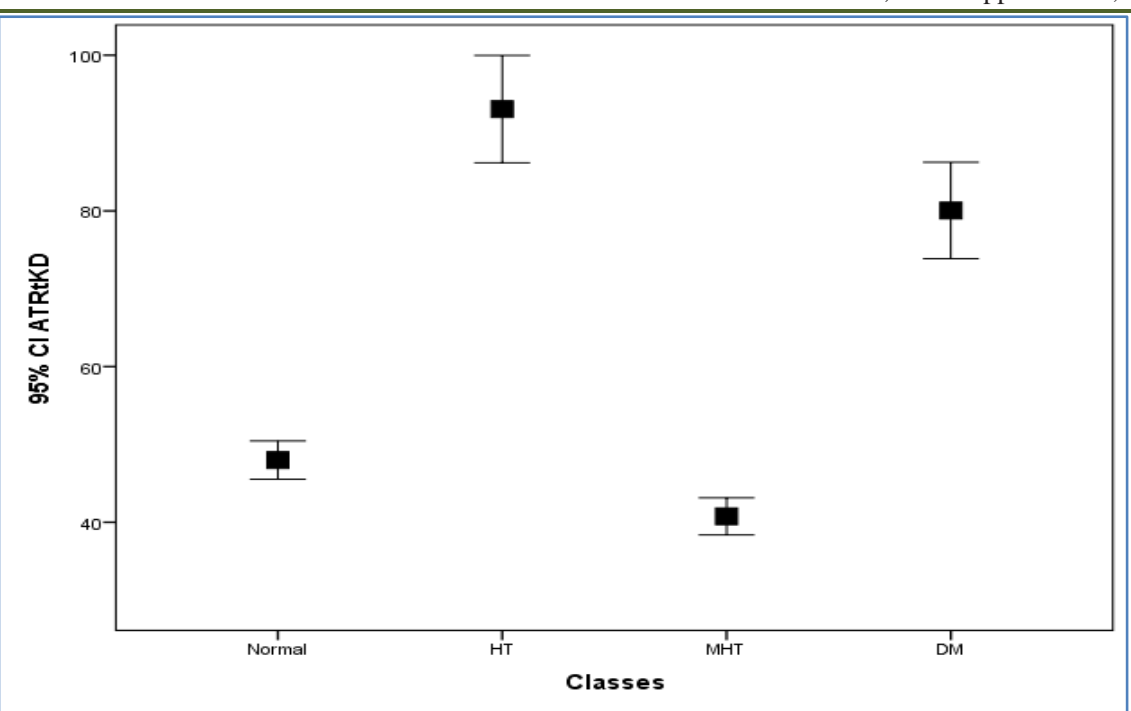

Fig-4: This figure shows the acceleration time of the rt kidney, which is low in normal patients, lower in mild hypertensive patients, while it is higher in hypertensive patients and little less in diabetic ones

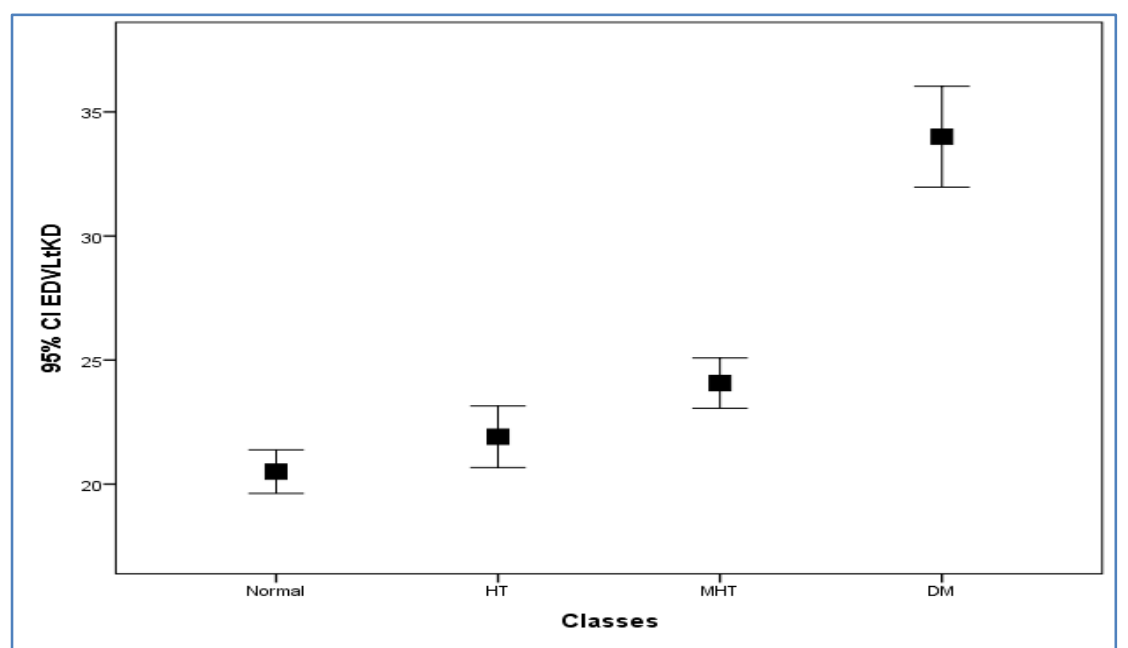

Fig-5: shows the end diastolic velocity of the left kidney in normal patients, hypertensive, mild hypertensive and diabetic patients. it is found to be high in diabetic patients, while it is very low in normal patents, a little bit more in hypertensive patients, and one more grad higher in mild hypertensive patients

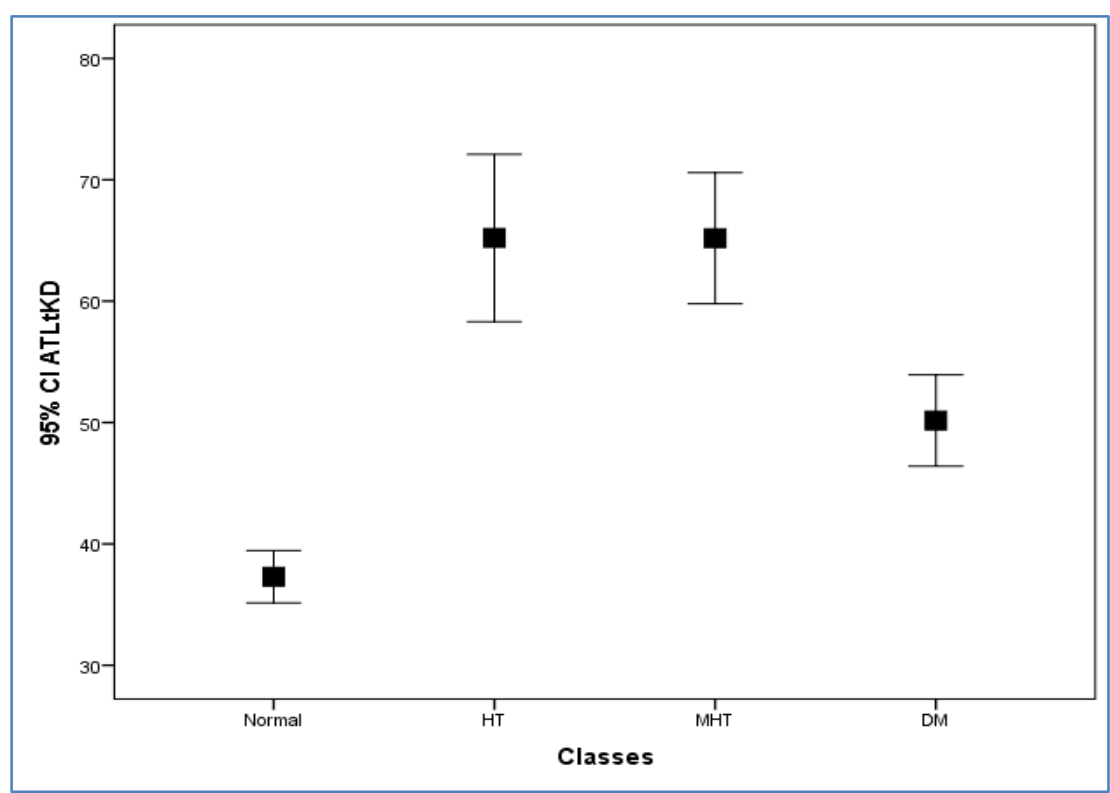

Fig-6: Shows the acceleration time of the left kidney in normal patients, hypertensive, mild hypertensive and diabetic ones 


\section{DisCUSSIONS}

Table 1. Show classification score matrix generated by linear discriminate analysis and the overall classification accuracy of renal disorders $95.4 \%$, were the classification accuracy of normal $98.6 \%$, HT 94\%, and MHT 93.8\%, while the DM showed a classification accuracy of $92.9 \%$.

Figure 1. Error bar plot shows the BMI of normal patients, hypertensive, mild hypertensive and diabetic patient's. The BMI is low in normal patients while it is high in hypertensive and diabetic patients and moderate in mild hypertensive patients.

Figure 2. This diagram shows the depth of the left kidney in normal patients, hypertensive, mild hypertensive and diabetic patients. The depth is medium in normal patients, while it is smaller in hypertensive patients; the depth is bigger in mild hypertensive patients and little less in diabetic patients,

Figure 3. Shows the end diastolic velocity of the right kidney in normal patients, hypertensive, mild hypertensive and diabetic patients. In normal patients the end diastolic is low, but lower in mild hypertensive patients, in diabetic patients it is higher than in .mild hypertensive patients, but less high than in hypertensive patients.

Figure 4. This figure shows the acceleration time of the rt kidney, which is low in normal patients, lower in mild hypertensive patients, while it is higher in hypertensive patients and little less in diabetic ones.

Figure 5. shows the end diastolic velocity of the left kidney in normal patients, hypertensive, mild hypertensive and diabetic patients. it is found to be high in diabetic patients, while it is very low in normal patents, a little bit more in hypertensive patients, and one more grad higher in mild hypertensive patients.

Figure 6. Shows the acceleration time of the left kidney in normal patients, hypertensive, mild hypertensive and diabetic ones.

It is very low in normal patients, high in hypertensive and mild hypertensive patients and little less in diabetic patients.

\section{CONCLUSION}

Characterization of kidney disease in adults' patients using ultrasonography, the data of this study was collected from 200 adults' patients both gender suffering from renal.

Classification score matrix generated by linear discriminate analysis and the overall classification accuracy of renal disorders $95.4 \%$, were the classification accuracy of normal 98.6\%, HT 94\%, and
MHT $93.8 \%$, while the DM showed a classification accuracy of $92.9 \%$.

Figure 1. Error bar plot shows the BMI of normal patients, hypertensive, mild hypertensive and diabetic patient's. The BMI is low in normal patients while it is high in hypertensive and diabetic patients and moderate in mild hypertensive patients.

The end diastolic velocity of the right kidney in normal patients, hypertensive, mild hypertensive and diabetic patients. In normal patients the end diastolic is low, but lower in mild hypertensive patients; in diabetic patients it is higher than in .mild hypertensive patients, but less high than in hypertensive patients. It is very low in normal patients, high in hypertensive and mild hypertensive patients and little less in diabetic patients.

\section{REFERNCES}

1. Fernbach S, Fernstein K. The kidney: normal renal anatomy, variants, and congenital anomalies. In: Bulas DI, Faerber EN, Adler BH. eds. Caffey's pediatric diagnostic imaging. Philadel phia, PA: Mosby-Elsevier. 2008:2234-2262

2. Geelhoed JJ, Kleyburg-Linkers VE, Snijders SP. Reliability of renal ultrasound measurements in children. Pediatr Nephrol. 2009; 24:1345-1353

3. Schlesinger AE, Hernandez RJ, Zerin JM, Marks TI, Kelsch RC. Interobserver and intraobserver variations in sonographic renal length measurements in children. AJR. 1991; 156:10291032

4. Zerin JM, Blane CE. Sonographic assessment of renal length in children: a reappraisal. Pediatr Radiol. 1994; 24:101-106

5. Leroy S, Chalumeau M, Ulinski T. Impressive renal damage after acute pyelonephritis in a child. Pediatr Nephrol. 2010; 25:1365-1368.

6. Roger SD, Beale AM, Cattell, WR and WEBB, JAW, What is the value of measuring renal parenchymal thicknessbeforerenalbiopsy? Clin. Radioi. 1994; 49: 45-49.

7. MADAIO, MP, Renalbiopsy, KidneyInt. 1990; 38,529-543 (1990).

8. Kidney Disease: Improving Global Outcomes (KDIGO) CKD Work Group. KDIGO 2012 clinical practice guideline for the evaluation and management of chronic kidney disease. Kidney Int Suppl. 2013; 3(1):1-150.

9. Centers for Disease Control and Prevention. Chronic kidney disease (CKD) surveillance system. 2017; https: //nccd.cdc.gov/CKD/data.aspx. Accessed January 20, 2017.

10. American Diabetes Association. 3. Comprehensive medical evaluation and assessment of comorbidities [published correction appears in Diabetes Care. 2017; 40(7): 985]. Diabetes Care. 2017; 40(suppl 1): S25-S32. 
Abdulrahman M. B. Arbab et al; Sch J App Med Sci, Mar, 2021; 9(3): 372-377

11. Moyer VA; U.S. Preventive Services Task Force. Screening for chronic kidney disease: U.S. Preventive Services Task Force recommendation statement. Ann Intern Med. 2012; 157(8): 567570.

12. Qaseem A, Hopkins RH Jr, Sweet DE, Starkey M, Shekelle P. Clinical Guidelines Committee of the American College of Physicians. Screening, monitoring, and treatment of stage 1 to 3 chronic kidney disease: a clinical practice guideline from the American College of Physicians. Ann Intern Med. 2013; 159 (12): 835-847.

13. American Academy of Family Physicians. Clinical practice guideline: chronic kidney disease. http: //www.aafp.org/patient-

care/clinicalrecommendations/all/chronic-kidneydisease.html. Accessed October
26, 2017.

14. Chau K, Hutton H, Levin A. Laboratory assessment of kidney disease: glomerular filtration rate, urinalysis, and proteinuria. In: Skorecki K, eds. Brenner \& Rector's The Kidney. 10th ed. Philadelphia, Pa.: Elsevier; 2016: 780-803.

15. Fan L, Inker LA, Rossert J. Glomerular filtration rate estimation using cystatin $\mathrm{C}$ alone or combined with creatinine as a confirmatory test. Nephrol Dial Transplant. 2014; 29(6): 1195 -1203.

16. Ninomiya $\mathrm{T}$, Perkovic $\mathrm{V}$, de Galan BE. ADVANCE Collaborative Group. Albuminuria and kidney function independently predict cardiovascular and renal outcomes in diabetes. $\mathrm{J}$ Am Soc Nephrol. 2009;20(8):1813-1821. 